Check for updates

Cite this: RSC Adv., 2019, 9, 5512

Received 12th January 2019

Accepted 7th February 2019

DOI: 10.1039/c9ra00276f

rsc.li/rsc-advances

\section{Co-delivery of paclitaxel and gemcitabine via a self- assembling nanoparticle for targeted treatment of breast cancer $\uparrow$}

\author{
Meng Lei, ${ }^{a}$ Sijia Sha, ${ }^{a}$ Xueyuan Wang, ${ }^{b}$ Jia Wang, ${ }^{d}$ Xiao Du, (D) *c Hang Miao, ${ }^{\text {a }}$ \\ Hui Zhou, ${ }^{\text {b }}$ Enhe Bai, ${ }^{\text {b Jingmiao Shi }}{ }^{d}$ and Yongqiang Zhu*b
}

\begin{abstract}
Multi-functional nanoparticles can be used to improve the treatment index and reduce side effects of antitumor drugs. Herein, we developed a kind of multi-functional and highly biocompatible nanoparticle (NP) loaded with folic acid (FA), paclitaxel (PTX) and gemcitabine (GEM) via self-assembly to target cancer cells. The transmission electron microscopy (TEM) results showed that multi-functional FA targeting nanoparticles (MF-FA NPS) exhibited spherical morphology and favorable structural stability in aqueous solution. In addition, NPs (MF-FA NPs and MF NPs) exhibited comparable proliferation inhibition to breast cancer cell 4T1 compared with the pure drug. In in vivo antitumor studies, NPs showed an obviously enhanced anti-tumor efficacy compared with the pure drug. Furthermore, MF-FA NPs displayed higher tumor growth inhibition than MF NPs due to the specific targeting of FA to cancer cells. Consequently, the novel MF-FA NPs could be used as a potential chemotherapeutic formulation for breast cancer therapy.
\end{abstract}

\section{Introduction}

As one of the most common types of cancer, breast cancer has been a primary threat to women's health. ${ }^{1}$ Among cytotoxic chemotherapeutic agents for advanced breast cancer patients, prior to anthracycline therapy, is gemcitabine (GEM). ${ }^{2}$ However, there are still some drawbacks of GEM treatment that compromise its applications. ${ }^{3}$ For example, GEM lacks selectivity towards cancer tissues and enters cancer and healthy cells indiscriminately, which led to severe side effects and narrow therapeutic windows. ${ }^{3}$ In addition, it was reported that approximately $90 \%$ of GEM would be rapidly decomposed with a short half-life of $32 \mathrm{~min}$ in blood circulation due to the deamination to produce an inactive $2^{\prime}, 2^{\prime}$-difluorodeoxyuridine. ${ }^{2}$ Furthermore, cancer cells tend to become drug resistant after prolonged treatment with GEM. ${ }^{4}$

Combination chemotherapy is an effective strategy for alleviating the above problems. ${ }^{5-8}$ In comparison with conventional single-agent treatment, combination therapy is capable of promoting synergistic effects of different drugs, enhancing

${ }^{a}$ College of Science, Nanjing Forestry University, No. 159 Longpan Road, Nanjing 210037, PR China

${ }^{b}$ College of Life Science, Nanjing Normal University, No. 1 Wenyuan Road, Nanjing 210046, PR China. E-mail: zhyqscu@hotmail.com

${ }^{c}$ Department of Pharmaceutics, School of Pharmacy, China Pharmaceutical University, Nanjing 210009, PR China. E-mail: duxiaojianai@126.com

'Jiangsu Chia Tai Fenghai Pharmaceutical Co. Ltd., No. 9 Weidi Road, Nanjing 210046, PR China

† Electronic supplementary information (ESI) available. See DOI: $10.1039 / \mathrm{c} 9 \mathrm{ra00276f}$ therapeutic selectivity and overcoming multidrug resistance via distinct mechanisms of actions. ${ }^{\text {9-11 }}$ However, combination chemotherapy also has its own drawbacks due to different pharmacokinetic properties of the drugs, which increases the difficulty to obtain the optimal dose and further causes more adverse side effects. ${ }^{12,13}$

To address this dilemma, nanocarrier-based drug delivery systems (nano-DDS) have drawn more and more attentions for promoting therapeutic efficacy and overcoming the pharmacokinetic limitation of anti-cancer drugs by improving the balance between their efficacy and toxicity. ${ }^{\mathbf{1 4}}$ Especially polymer-drug conjugate, also known as polymeric prodrug, is the most investigated carriers and has demonstrated significant potentials in nanomedicine for their advanced drug delivery to the targets, by improving drug bioavailability, balancing the pharmacokinetics of hydrophilic and hydrophobic drugs, allowing the tumor-specific activation of drugs. ${ }^{15-19}$ Therefore, polymeric prodrugs provide a potential platform for effective anticancer drug delivery by synchronizing the advantages of polymeric prodrug and co-delivery systems, and are capable of achieving synergistic therapeutic effects through simultaneous multidrug accumulation..$^{20-23}$ Nevertheless, developing such a drug delivery system still faces technical challenges such as lacks of specific drug target and rapid intracellular drug release at the target site. ${ }^{24}$

Therefore, introduction of different biological ligands or antibodies into novel drug delivery system would be beneficial for selective delivery of chemotherapeutics to tumor cells. ${ }^{25}$ Folic acid (FA) is regarded as an important and active targeting agent, which shows a strong affinity for specific receptors that 
are uniquely overexpressed in tumor cells. ${ }^{26,27}$ Thus, introduction of FA onto polymer-drug conjugate would increase the drug accumulation at the tumor sites and promote cancer cell uptake via a folate receptor-mediated endocytosis, thereby improving drug bioavailability and enhancing the therapeutic of cancer treatments. ${ }^{28,29}$

In this manuscript, we developed a folate targeting multifunctionalized nanocarrier delivery system for co-delivery of hydrophilic chemotherapeutic drug GEM and hydrophobic PTX for the breast cancer treatment. The novel polymeric prodrug nanoparticles were constructed through electrostatic interactions using synthesized poly glutamic acid (PGA) conjugated GEM (PGA-GEM), PGA conjugated PTX (PGA-PTX), PGA conjugated FA (PGA-FA) and poly-lysine dendrimer (PLD/P-6). The two anti-cancer drugs and targeting ligand FA were all coupled to the polymer backbone of PGA through hydrolysable ester bond linkers. PLD was introduced as a cationic and biodegradable dendrimer to promote the self-assembly of polymeric prodrug and further increase the stability of multifunctionalized nanoparticles. In addition, PLD would also facilitate the drugs to escape from lysosome via the proton sponge effect to increase therapeutic efficacy. ${ }^{30}$ As illustrated in Fig. 1, the nanoparticles would preferentially accumulate at tumor tissue by passive targeting effect and enter tumor cells via folate receptor mediated endocytosis. Once the polymer enters the cells, ester bond of the polymer would be rapidly cleaved in response to the acidified endosomes and GEM and PTX would be sufficiently released, thereby leading to synergistic anticancer effects.

\section{Materials and methods}

\subsection{Materials}

1-Hydroxybenzotriazole (HOBT) was purchased from Accela ChemBio Co., Ltd (Shanghai, China). 1-(3-Dimethylaminopropyl)3-ethylcarbodiimide hydrochloride (EDCI), folic acid hydrate (FA), $N$-hydroxysuccinimide (NHS), 1,6-diaminohexane, L-glutamic acid (Glu), ethyldiisopropylamine (DIPEA) and trifluoroacetic acid (TFA) were obtained from Energy Chemical Technology Co., Ltd (Shanghai, China). Tert-butyl methyl ether was purchased from Shanghai Titan Scientific Co., Ltd (Shanghai, China). Boc-Lys,

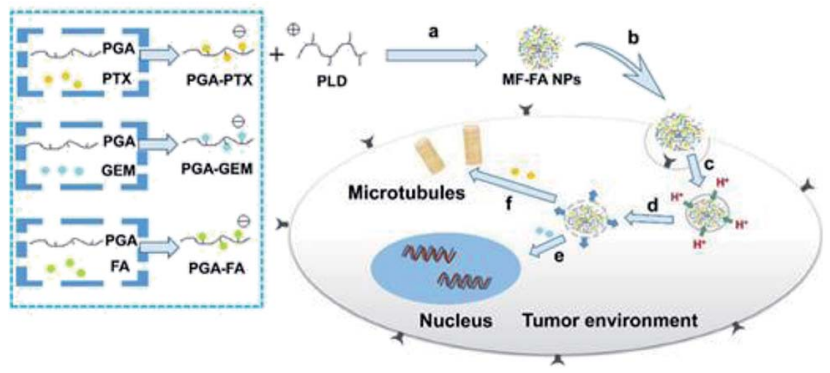

Fig. 1 Schematic illustration of the preparation of MF-FA NPs and the activated release behavior of PTX and GEM in tumor cells. (a) Selfassembly through electrostatic interaction. (b) Endocytosis. (c) Taken up by lysosomes. (d) Endosomal escape by proton influx. (e) GEM inhibits DNA replication. (f) PTX breaks down microtubules.
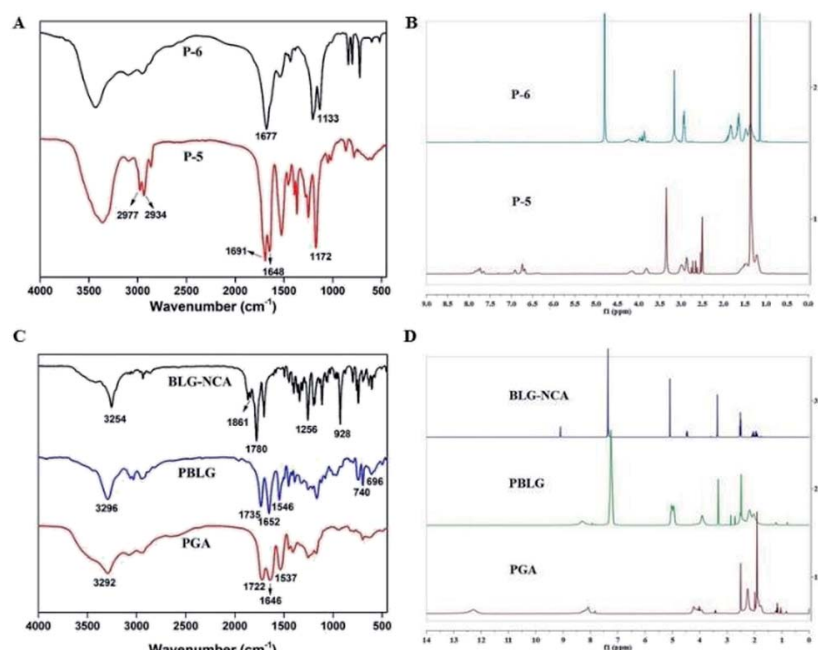

Fig. 2 Characterization of the compounds. (A) FT-IR spectra of P-5 and P-6. (B) ${ }^{1} \mathrm{H}$ NMR spectra of P-5 and P-6. (C) FT-IR spectra of BLGNCA, PBLG and PGA. (D) ${ }^{1} H$ NMR spectra of BLG-NCA, PBLG and PGA.

paclitaxel (PTX) and gemcitabine (GEM) were purchased from Bide Pharmatech Ltd (Shanghai, China).

\subsection{Synthesis of polymer-drug conjugates}

2.2.1 Synthesis of poly-lysine dendrimer (PLD/P-6). The synthetic route of the poly-lysine dendrimer (PLD/P-6) was shown in Fig. S1.† Boc-Lys (1.00 g, $1.89 \mathrm{mmol})$ and HOBT $(0.64 \mathrm{~g}, 4.74 \mathrm{mmol})$ were dissolved in DCM $(50 \mathrm{~mL})$. The mixture was stirred at $-10{ }^{\circ} \mathrm{C}$ for $10 \mathrm{~min}$, followed by adding EDCI (0.91 g, $4.74 \mathrm{mmol}$ ) and 1,6-hexanediamine (0.11 g, $0.95 \mathrm{mmol})$ into the system and stirred for another $30 \mathrm{~min}$. Subsequently, DIPEA $(0.73 \mathrm{~g}, 5.67 \mathrm{mmol})$ was added and the reaction was allowed to proceed for overnight under $\mathrm{N}_{2}$ atmosphere. Afterwards, the system was washed with $\mathrm{HCl}$ solution $(20 \mathrm{~mL} \times 2)$, $5 \% \mathrm{NaHCO}_{3}(20 \mathrm{~mL} \times 2)$, saturated sodium chloride solution and further dried over anhydrous $\mathrm{Na}_{2} \mathrm{SO}_{4}$. Then the solution was concentrated under vacuum to give compound $\mathrm{P}-1$. To remove the protecting group Boc, P-1 (0.85 g, $1.10 \mathrm{mmol})$ was dissolved in $5 \mathrm{~mL}$ DCM, followed by adding of trifluoroacetic acid (TFA) (1.23 mL, $16.50 \mathrm{mmol}$ ). The mixture was reacted for $3 \mathrm{~h}$, and the organic solvent was evaporated in vacuum and the residue was washed with methyl tert-butyl ether to give product $\mathrm{P}-2$. The stepwise growth of Lys was repeated to yield P-5, the protecting group Boc of which was removed to give PLD/P-6. P-5: ${ }^{1} \mathrm{H}$ NMR (400 MHz, DMSO-d ${ }_{6}$ ) $\delta$ 7.98-7.59 (-CONH), 4.16-3.82 $(-\mathrm{CH}), 2.90\left(-\mathrm{CH}_{2}\right), 1.48\left(-\mathrm{CH}_{2}\right), 1.36\left(-\mathrm{CH}_{3}\right), 1.33-1.28\left(-\mathrm{CH}_{2}\right)$, $1.22\left(-\mathrm{CH}_{2}\right)$. FTIR peak $\left(\mathrm{cm}^{-1}\right): 2997,2934,1691,1648,1172 . \mathrm{P}-$ 6: ${ }^{1} \mathrm{H}$ NMR $\left(400 \mathrm{MHz}, \mathrm{D}_{2} \mathrm{O}\right) \delta$ 4.04-3.82 (-CH), 3.31-3.02 $\left(-\mathrm{CH}_{2}\right)$, 3.00-2.84 $\left(-\mathrm{CH}_{2}\right), 2.05-1.69\left(-\mathrm{CH}_{2}\right), 1.66-1.43\left(-\mathrm{CH}_{2}\right), 1.40-1.11$ $\left(-\mathrm{CH}_{2}\right)$. FTIR peak $\left(\mathrm{cm}^{-1}\right): 1677,1133$.

The average molecular weight of P-6 was determined by gel permeation chromatography (PL GPC 50). The dissolution solvent was water and mobile phase was composed of $0.2 \mathrm{M}$ $\mathrm{NaNO}_{2}$. GPC was performed on a column $(2 \times \mathrm{PL}$ aquagel-OH $308 \mu \mathrm{m}, 7.5 \times 300 \mathrm{~mm}$ ) at a $1.0 \mathrm{~mL} \min ^{-1}$ flow rate with a refractive index detector (sample concentration: $0.10 \mathrm{mg}$ 
$\mathrm{mL}^{-1}$, injection volume: $100.0 \mu \mathrm{L}$ ). The average molecular weight (2403 Da) and polydispersity index (1.08) were calculated from the GPC retention time $(17.33 \mathrm{~min}, y=11.104568-$ $0.445890 x)$.

2.2.2 Synthesis of poly glutamic acid (PGA). The synthetic route of the poly glutamic acid (PGA) was shown in Fig. S2. $\dagger$ In brief, Glu (Z) (5.00 g, $211.00 \mathrm{mmol})$ was dissolved in anhydrous THF (100 mL), then triphosgen $(6.25 \mathrm{~g}, 21.06 \mathrm{mmol})$ was added and the system took place at $50{ }^{\circ} \mathrm{C}$ for $3 \mathrm{~h}$ under $\mathrm{N}_{2}$ atmosphere. After removing the remaining organic solvent in vacuum, the residue was washed with cold $n$-hexane to obtain purified BLGNCA. The average molecular weight was determined by gel permeation chromatography (PL GPC 50) (sample concentration: $0.10 \mathrm{mg} \mathrm{mL} \mathrm{m}^{-1}$, injection volume: $\left.100.0 \mu \mathrm{L}\right)$. The average molecular weight (6425 Da) and polydispersity index (1.07) were calculated from the GPC retention time (17.13 $\mathrm{min}, y=$ $11.104568-0.445890 x) .{ }^{1} \mathrm{H}$ NMR $\left(400 \mathrm{MHz}, \mathrm{DMSO}_{6}\right) \delta 7.37-$ $7.30(-\mathrm{Ph}), 5.08\left(-\mathrm{COOCH}_{2}\right), 4.47(-\mathrm{CHNH}), 2.61-2.44\left(-\mathrm{CH}_{2}\right)$, 2.08-1.85 $\left(-\mathrm{CH}_{2}\right)$. FTIR peak $\left(\mathrm{cm}^{-1}\right)$ : 3254, 1861, 1780, 928.

For synthesis of PBLG, BLG-NCA (4.97 g, $18.89 \mathrm{mmol}$ ) and $\mathrm{N}$ hexylamine $(25.48 \mathrm{mg}, 0.25 \mathrm{mmol}$ ) were dissolved in DMF (25 $\mathrm{mL}$ ) in a $100 \mathrm{~mL}$ round-bottom eggplant flask. The mixture reaction was reacted at $35{ }^{\circ} \mathrm{C}$ for $72 \mathrm{~h}$. Subsequently, the reaction mixture was poured into $500 \mathrm{~mL}$ of cold methyl tert-butyl ether, and the precipitate was dried in vacuum and white powder PBLG was obtained. ${ }^{1} \mathrm{H}$ NMR (400 MHz, DMSO-d 6 ) $\delta 7.94(-\mathrm{CONH}), 7.29(-\mathrm{Ph}), 5.20-4.79\left(-\mathrm{CH}_{2}\right), 3.91(-\mathrm{CH}), 3.04$ $\left(-\mathrm{CH}_{2}\right), 2.18\left(-\mathrm{CH}_{2}\right), 2.03\left(-\mathrm{CH}_{2}\right), 1.40\left(-\mathrm{CH}_{2}\right), 1.21\left(-\mathrm{CH}_{2}\right), 0.80$ $\left(-\mathrm{CH}_{3}\right)$. FTIR peak $\left(\mathrm{cm}^{-1}\right): 3296,1652,1546,740,696$.

In order to prepare PGA, PBLG $(5.00 \mathrm{~g}, 22.62 \mathrm{mmol})$ was dissolved in $\mathrm{MeOH}(15 \mathrm{~mL})$. Then $3.6 \mathrm{~g}$ of $\mathrm{NaOH}(90.00 \mathrm{mmol})$ was dissolved in $\mathrm{H}_{2} \mathrm{O}(2 \mathrm{~mL})$ and injected into the mixture. The mixture was stirred for $5 \mathrm{~h}$ at room temperature. Then $30 \mathrm{~mL}$ of EtOH was poured into the system and centrifuged at 6000 $\mathrm{rpm} \min ^{-1}$ for $10 \mathrm{~min}$ to separate precipitate, which was further washed for several times until the $\mathrm{pH}$ was 7-8. Subsequently, the dried precipitate was dissolved in $5 \mathrm{~mL}$ of $\mathrm{HCl} / \mathrm{EA}$ solution. After 10 min of stirring, the remaining solvent was removed to obtain PGA. ${ }^{1} \mathrm{H}$ NMR (400 MHz, DMSO-d 6$) \delta 12.28(-\mathrm{COOH})$, $8.13(-\mathrm{CONH}), 4.27-3.82(-\mathrm{CH}), 3.43\left(-\mathrm{CH}_{2}\right), 2.25\left(-\mathrm{CH}_{2}\right), 2.00-$ $1.68\left(-\mathrm{CH}_{2}\right), 1.37\left(-\mathrm{CH}_{2}\right), 1.23\left(-\mathrm{CH}_{2}\right), 0.84\left(-\mathrm{CH}_{3}\right)$. FTIR peak $\left(\mathrm{cm}^{-1}\right): 3292,1722,1646,1537$.

2.2.3 Preparation of PGA-PTX and PGA-GEM. PGA $(0.40 \mathrm{~g}$, $3.30 \mathrm{mmol}$ ) was dissolved in $5 \mathrm{~mL}$ of DMSO, followed by adding EDCI $(0.30 \mathrm{~g}, 1.56 \mathrm{mmol})$ and PTX $(60.00 \mathrm{mg}, 0.07 \mathrm{mmol})$ to the mixture. The system was stirred at room temperature overnight and the product was dialyzed against deionized water with cellulose tubing (MWCO: $1000 \mathrm{Da}$ ), followed by lyophilization to obtain PGA-PTX. ${ }^{1} \mathrm{H}$ NMR (400 MHz, DMSO- $\left.d_{6}\right) \delta$ 7.89-7.68 (-Ph-d), 7.33 (-Ph-c), 4.49 (-COCHNH-b), 1.02 (-CH $\mathrm{CH}_{3}$-a) (Fig. 3B). FTIR peak $\left(\mathrm{cm}^{-1}\right)$ : 3066, 768, 705.

For synthesis of PGA-GEM, PTX was replaced by GEM, and the preparation was similar to that of PGA-PTX. ${ }^{1} \mathrm{H}$ NMR $(400 \mathrm{MHz}$, DMSO- $d_{6}$ ) $\delta 7.45$ (-Ph-f), 5.99 (- $\left.\mathrm{CF}_{2} \mathrm{CHO}-\mathrm{e}\right), 4.49$ (-COCHNH-b), $0.97\left(-\mathrm{CH}_{3}\right.$-a) (Fig. 3D). FTIR peak $\left(\mathrm{cm}^{-1}\right)$ : 1209, 1130.

2.2.4 Synthesis of PGA-FA. The synthetic route of the modified PGA with FA was displayed in Fig. S3. $\dagger$ Generally, FA
(1.07 g, $2.41 \mathrm{mmol}$ ) was dispersed in $20 \mathrm{~mL}$ of DMSO, then NHS (277.00 $\mathrm{mg}, 2.41 \mathrm{mmol}$ ) and EDCI (694.00 $\mathrm{mg}, 3.60 \mathrm{mmol}$ ) were added and the resulting solution was stirred for $1 \mathrm{~h}$ at room temperature. Afterwards, $0.5 \mathrm{~mL}$ of $\mathrm{Et}_{3} \mathrm{~N}$ was added into the solution and stirred for another $36 \mathrm{~h}$ in the dark. The reaction system was poured into a mixture of methyl tert-butyl ether: acetone $(40 \mathrm{~mL} ; 7: 3, \mathrm{v} / \mathrm{v})$, and the precipitate was dried in vacuum and FA-NHS was obtained. Subsequently, FA-NHS (200.00 $\mathrm{mg}, 0.37 \mathrm{mmol}$ ) was dissolved in $8 \mathrm{~mL}$ of DMSO, then $\mathrm{Et}_{3} \mathrm{~N}$ (151.90 $\mathrm{mg}, 0.56 \mathrm{mmol}$ ) and 3-aminopropanol (27.90 mg, $0.37 \mathrm{mmol}$ ) were added respectively. The mixture was stirred for $24 \mathrm{~h}$ in the dark at room temperature. The system was poured into a mixture of methyl tert-butyl ether : acetone $(20 \mathrm{~mL} ; 7: 3$, $\mathrm{v} / \mathrm{v}$ ), and the precipitate was dried in vacuum and light yellow product FA-OH was obtained. For the preparation of PGA-FA, PGA (200.00 mg, $1.65 \mathrm{mmol}$ ) was dispersed in $4 \mathrm{~mL}$ of DMSO, followed by adding EDCI (316.86 $\mathrm{mg}, 1.65 \mathrm{mmol}$ ) and DMAP (201.95 $\mathrm{mg}, 1.65 \mathrm{mmol}$ ) respectively. The mixture was stirred for $30 \mathrm{~min}$, then FA-OH $(66.00 \mathrm{mg}, 0.13 \mathrm{mmol})$ was added and further stirred for $24 \mathrm{~h}$. The mixture was then dialyzed against deionized water with cellulose tubing (MWCO: $1000 \mathrm{Da}$ ), followed by lyophilization to obtain PGA-FA. The ${ }^{1} \mathrm{H}$ NMR and FTIR spectra of PGA-FA were as follows: ${ }^{1} \mathrm{H}$ NMR $(400 \mathrm{MHz}$, DMSO- $\left.d_{6}\right) \delta 7.48$ (-Ph-h), 6.66 (-Ph-g), 4.49 (-COCHNH-b), 0.94 $\left(-\mathrm{CH}_{3}\right.$-a) (Fig. 3F). FTIR peak $\left(\mathrm{cm}^{-1}\right): 3117,1410,1131,833,763$.

\subsection{Preparation and characterization of functionalized nanoparticles}

Multi-functional FA targeting nanoparticles (MF-FA NPs) were prepared by electrostatic interaction between positively charged

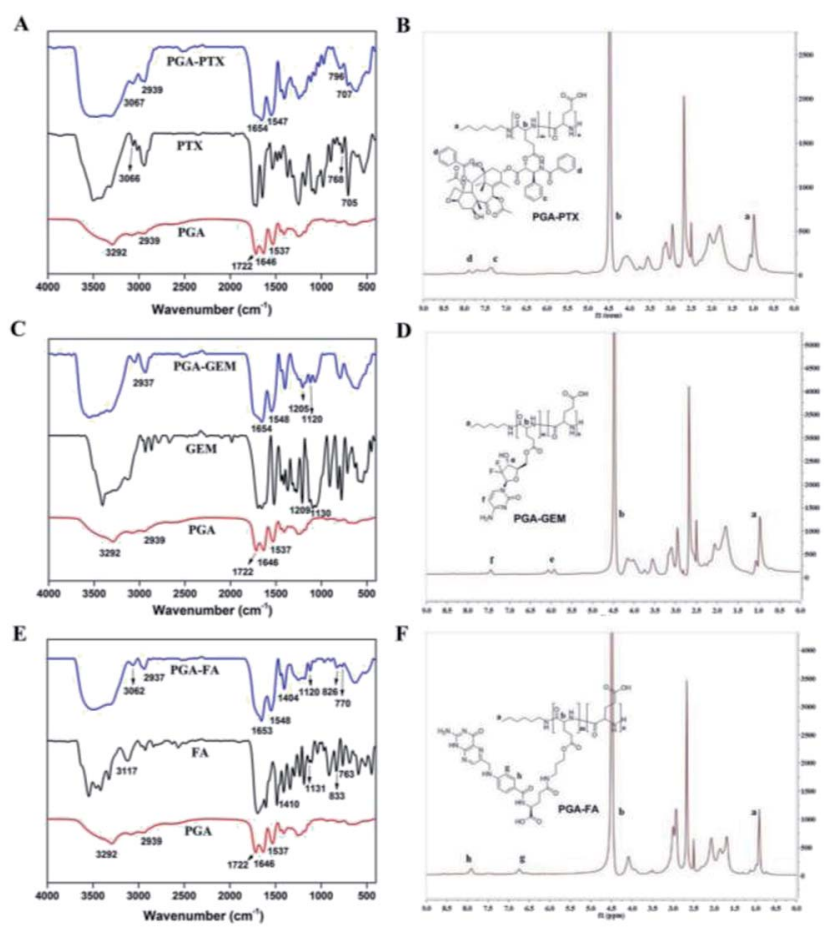

Fig. 3 FT-IR spectra and ${ }^{1} \mathrm{H}$ NMR spectra of PGA-PTX, PGA-GEM and PGA-FA. 
P-6 and negatively charged PGA-PTX, PGA-GEM and PGA-FA. In brief, $5.00 \mathrm{mg}$ of PGA-PTX, PGA-GEM and PGA-FA was dissolved in $10 \mathrm{~mL}$ of distilled water, respectively. Then $300 \mu \mathrm{L}$ of each sample was fully mixed by vortex. Subsequently, different ratio of P- 6 solution was added to the above mixed solution, and further were incubated at $4{ }^{\circ} \mathrm{C}$ for $3 \mathrm{~h}$ to obtain the nanoparticles.

Particle size distribution and zeta potential were measured using a Malvern Zetasizer 3000 system (Malvern Instruments Ltd., UK). In order to observe the morphology of NPs, the samples were obtained by depositing the nanoparticles solution onto double carbon coated copper grid and drying at room temperature. Transmission electron microscopy (TEM) was applied to observe morphology of nanoparticle samples.

\subsection{Determination of drug loading}

Different concentrations of PTX, GEM and FA were prepared and measured by UV-Vis spectrophotometer to get standard curve. The absorbance of PTX, GEM and FA which were encapsulated in PGA-PTX, PGA-GEM and PGA-FA were determined by UV-Vis spectrophotometer at $230 \mathrm{~nm}, 269 \mathrm{~nm}$ and $280 \mathrm{~nm}$, respectively. The drug concentration of PGA-PTX, PGAGEM and PGA-FA were calculated by the standard curve. The drug loading content was calculated by the following equation:

$$
\mathrm{DL}(\%)=\frac{\text { The concentration of the drug in the sample }}{\text { The concentration of the sample }} \times 100 \%
$$

\subsection{In vitro drug release of PTX and GEM}

The drug release profiles of PTX and GEM were investigated by the dialysis bag diffusion technique. Briefly, $2 \mathrm{~mL}$ of MF-FA NPs solution (1.5 mg PTX and $1.5 \mathrm{mg}$ GEM) was placed in a dialysis bag $(\mathrm{MWCO}=3500 \mathrm{Da})$ and immersed in a $30 \mathrm{~mL}$ of PBS buffer solution containing $0.5 \%$ Tween 80 at $\mathrm{pH} 5.5$ and $\mathrm{pH}$ 7.4. The released solution was kept at $37{ }^{\circ} \mathrm{C}$ with shaking at $100 \mathrm{rpm}$. At predetermined times, $1 \mathrm{~mL}$ of samples were withdrawn and replaced with an equivalent volume of fresh medium. The concentration of PTX and GEM was analyzed by UltraPerformance Liquid Chromatography (UPLC). The mobile phase was composed of DPBS/ACN $(50: 50, \mathrm{v} / \mathrm{v})$ with a flow rate

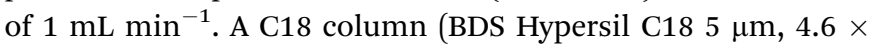
$150 \mathrm{~mm}$ column) was used in UPLC analysis where PTX was detected at $230 \mathrm{~nm}$ and GEM was detected at $269 \mathrm{~nm}$ (PTX: $0.93 \min , y=0.2191 x-0.2455, R^{2}=0.998$; GEM: $5.94 \mathrm{~min}, y=$ $\left.0.2883 x-0.0252, R^{2}=0.998\right)$, respectively.

\subsection{Stability of nanoparticles}

The stability of MF-FA NPs was evaluated by monitoring changes in particle size, PDI and zeta potential in distilled water. MF-FA NPs solution $\left(0.5 \mathrm{mg} \mathrm{mL}^{-1}\right)$ was kept at room temperature for $96 \mathrm{~h}$, and at prearranged intervals the parameters were determined.

\subsection{Hemolysis study}

Hemolysis study was investigated using Micro free hemoglobin kit (Nanjing Jian Cheng Bioengineering Institute). Firstly, fresh whole blood was collected from healthy mice, and was diluted in normal saline to separate red blood cell (RBC) from the whole blood through centrifugation at $2000 \mathrm{rpm}$ for 10 minutes at $4{ }^{\circ} \mathrm{C}$, which was then washed three times with normal saline and further diluted with saline to obtain a $2 \%$ RBC suspension $(\mathrm{v} / \mathrm{v})$. Subsequently, the red blood cell suspension was treated with MF-FA NPs and MF NPs, distilled water and saline, respectively..$^{31}$ They were incubated at $37{ }^{\circ} \mathrm{C}$ for $1 \mathrm{~h}$ and centrifuged at $2000 \mathrm{rpm}$ for 10 minutes to obtain the supernatant. Standard operating procedures of the assay by the kit manufacturer were followed and the data was analyzed at $510 \mathrm{~nm}$ by UV-Vis spectrophotometer (UV-2550, Kyoto, Japan).

\subsection{Study of antiproliferative ability}

The antiproliferative ability of drug-loaded nanoparticles and blank nanoparticles was evaluated by the Cell Counting Kit-8 (CCK-8) assay. The breast cancer cell line 4T1 was provided by Shanghai Cell Institute (Shanghai, China). The cells were cultured in RPMI 1640 with 10\% fetal bovine serum (FBS), $100 \mathrm{U}$ $\mathrm{mL}^{-1}$ penicillin and $100 \mathrm{U} \mathrm{mL}^{-1}$ streptomycin, and incubated at $37{ }^{\circ} \mathrm{C}$ in $5 \% \mathrm{CO}_{2}$. Then cells were seeded in 96-well plates at a density of $5 \times 10^{3}$ cells per well and cultured for $24 \mathrm{~h}$. The cellular uptake of drugs via receptor-mediated endocytosis occurred in a short time period. Then serial dilutions of GEM and PTX were maintained in all cases: MF-FA NPs, MF NPs and pure drug (PTX : GEM = $1: 1$ ). After further incubation for up to $72 \mathrm{~h}, 10 \mu \mathrm{L}$ of CCK-8 solution was added to each well and incubated for $3 \mathrm{~h}$. Cell viability was calculated by a microplate reader (BMG CLARIO star, German) at $450 \mathrm{~nm}$.

\subsection{In vivo efficacy study}

BALB/c mice ( $20 \pm 2 \mathrm{~g}, 6-8$ weeks old, female) were obtained from Shanghai SIPPE-BK laboratory animal Co., Ltd (Shanghai, China) and acclimatized for 7 days after arrival. The mice were housed in cages with free access to food and water. All animal procedures were performed in accordance with the Guidelines for Care and Use of Laboratory Animals of Nanjing Normal University, and the animal use protocol was approved by the ethics committee of Nanjing Normal University. Tumor-bearing $\mathrm{BALB} / \mathrm{c}$ mice were prepared by injection of $1 \times 10^{6} 4 \mathrm{~T} 1$ cells per mouse in the left armpit. When the tumor volume reached approximately $80 \mathrm{~mm}^{3}$, mice were randomly divided into five groups with three mice in each group and intravenously administered with saline, pure drug (PD), MF-FA NPs, MF NPs, respectively. Saline was used as a negative control, and pure drug as a positive control. The group of pure drug exhibited serious toxicity over the concentration of $5 \mathrm{mg} \mathrm{kg}^{-1}$. Nanogroups showed good biocompatibility at the concentration of $10 \mathrm{mg} \mathrm{kg}^{-1}$ due to slow release of drugs. So nano-groups and pure drug group were injected via the tail vein at a dose of $10 \mathrm{mg}$ $\mathrm{kg}^{-1}$ and $5 \mathrm{mg} \mathrm{kg}^{-1}$ respectively every other day. 
All mice were tagged and the tumor volume was calculated according to the formula of $\left(a \times b^{2}\right) / 2$, where " $a$ " was the major axis and " $b$ " was the minor axis. At the same time, the body weight of the mice was also monitored. Treatment proceeded for 21 days. After day 21, the mice were sacrificed and tumors were excised.

\subsection{Statistical analysis}

At least three replicates were used in the study, and data were expressed as mean \pm standard deviation (SD). Statistical evaluation between different groups was analyzed by Student's $t$-test or one-way ANOVA. $P<0.05$ indicated statistical significance in experiments.

\section{Results and discussion}

\subsection{Synthesis and characterization of polymer-drug conjugates}

3.1.1 Synthesis and characterization of P-6. P-6 was formed by the gradual reaction of 1,6-hexanediamine as a core under the homogeneous conditions. Firstly, 1,6-hexanediamine reacted with Boc-Lys under amide condensation and P-1 was obtained. Then the removal of Boc group of P-1 using trifluoroacetic acid (TFA) gave P-2. The stepwise growth of Lys was repeated to yield P-6. The structure of compounds were confirmed by FT-IR (Bruker, VERTEX 80V, Germany) and ${ }^{1} \mathrm{H}$ NMR spectra (Varian INOVA $400 \mathrm{MHZ}$ nuclear magnetic resonance instrument).

In the IR spectrum of P-5 (Fig. 2A), the stretching vibrations of C-H on tert-butyl were observed at $2977 \mathrm{~cm}^{-1}$ and $2934 \mathrm{~cm}^{-1}$. Peaks at $1691 \mathrm{~cm}^{-1}$ and $1648 \mathrm{~cm}^{-1}$ could be attributed to the $\mathrm{C}=\mathrm{O}$ on the Boc group. In the IR spectrum of P-6, the intensity of four peaks above were significantly reduced and the peak at $1133 \mathrm{~cm}^{-1}$ corresponded to stretching vibration of primary amine C-N. Additionally, in the ${ }^{1} \mathrm{H}$ NMR spectra of P-5 and P-6 (Fig. 2B), the disappearance of hydrogen of P-6 at $1.36 \mathrm{ppm}$ $\left(-\mathrm{CH}_{3}\right)$ indicated the successful removal of the Boc group. All the data confirmed the structure of P-6.

3.1.2 Synthesis and characterization of PGA. For synthesis of PGA, BLG-NCA was prepared using Glu (Z) with triphosgen. Then, PBLG was polymerized by ring opening from BLG-NCA using $N$-hexylamine as a nucleophilic initiator. PBLG reacted with $\mathrm{NaOH}$ to remove benzyl and PGA was obtained.

As shown in Fig. 2C, in the IR spectrum of BLG-NCA, the observed absorbance bands at $1861 \mathrm{~cm}^{-1}, 1780 \mathrm{~cm}^{-1}$ and $1256 \mathrm{~cm}^{-1}$ were characterized for the stretching vibrations of the two $\mathrm{C}=\mathrm{O}$. The peak at $928 \mathrm{~cm}^{-1}$ corresponded to stretching vibrations of $\mathrm{O}=\mathrm{C}-\mathrm{O}-\mathrm{C}=\mathrm{O}$. The results indicated successful synthesis of BLG-NCA. In the IR spectrum of PBLG, the disappearance of characteristic absorption peaks of BLG-NCA (1861 $\mathrm{cm}^{-1}, 1780 \mathrm{~cm}^{-1}, 1256 \mathrm{~cm}^{-1}$ and $928 \mathrm{~cm}^{-1}$ ) indicated that the BLG-NCA monomer was completely polymerized. The peak at $3296 \mathrm{~cm}^{-1}$ and $1652 \mathrm{~cm}^{-1}$ were the stretching vibration absorption peak of amino group and carbonyl in the amide respectively. The peak at $1546 \mathrm{~cm}^{-1}$ was assigned to bending vibration of $\mathrm{N}-\mathrm{H}$ in amide and $\mathrm{C}-\mathrm{N}$ stretching vibration, which indicated that the BLG-NCA monomer was bonded with peptide to form PBLG. The peaks at $740 \mathrm{~cm}^{-1}$ and $696 \mathrm{~cm}^{-1}$ were attributed to the deformation vibration of benzene ring. In the IR spectrum of PGA, the disappearance of $740 \mathrm{~cm}^{-1}$ and $696 \mathrm{~cm}^{-1}$ indicated that benzyl group was removed. Therefore, the results indicated successful synthesis of PGA.

Structures of BLG-NCA, PBLG and PGA were further confirmed by ${ }^{1} \mathrm{H}$ NMR, which were showed in Fig. 2D. For the BLG-NCA, $2.0 \mathrm{ppm}$ and $2.45 \mathrm{ppm}$ were related to $-\mathrm{CH}_{2}$ and $4.47 \mathrm{ppm}$ was related to $-\mathrm{CHNH}$. Signal at $5.08 \mathrm{ppm}$ was methylene peak of $-\mathrm{CH}_{2} \mathrm{Ph}$ and $7.3 \mathrm{ppm}$ was the characteristic of $-\mathrm{Ph}$ peak. The characteristic of $-\mathrm{Ph}$ peak at $7.29 \mathrm{ppm}$ and methylene peak of benzyl at $5.2 \mathrm{ppm}$ can be found in the ${ }^{1} \mathrm{H}$ NMR spectrum of PBLG. In PGA, both the disappearance of these two peaks and the appearance peak of $-\mathrm{COOH}$ in 12.28 ppm verified its structure. The degree of polymerization (50) of PBLG was quantified from the peak integration ratio of hexylamine at $0.80 \mathrm{ppm}\left(-\mathrm{CH}_{3}\right)$ to the $\alpha$-carbon proton at $3.91 \mathrm{ppm}(-\mathrm{CH})$ of PBLG. Therefore, the results indicated successful synthesis of PGA.

3.1.3 Synthesis and characterization of PGA-PTX, PGAGEM and PGA-FA. PGA reacted with GEM and PTX through dehydration condensation. Additionally, FA-OH was synthesized by 3-aminopropanol and the active hydroxylic group of FA. The carboxyl groups of PGA were conjugated with the hydroxyl group of FA-OH via esterification and gave the PGA-FA.

As shown in Fig. 3A, in FT-IR spectra of PTX, the observed absorbance bands at $705 \mathrm{~cm}^{-1}, 768 \mathrm{~cm}^{-1}$ and $3066 \mathrm{~cm}^{-1}$ were characteristics for the bending vibration of $\mathrm{C}-\mathrm{H}$ on benzene ring. The bending vibration at $1646 \mathrm{~cm}^{-1}$ and $1537 \mathrm{~cm}^{-1}$ was attributed to the $\mathrm{C}=\mathrm{O}$ and $\mathrm{N}-\mathrm{H}$ in PGA, respectively. The observed absorbance bands at $3292 \mathrm{~cm}^{-1}$ and $1722 \mathrm{~cm}^{-1}$ were characteristics for the stretching vibration of $-\mathrm{COOH}$ in PGA. The peak at $2939 \mathrm{~cm}^{-1}$ was attributed to the stretch absorption of $-\mathrm{CH}_{2}$ in PGA and PGA-PTX. The appearance of the above characteristic peaks in PGA-PTX proved the structure of PGAPTX. IR spectrum of PGA-GEM was shown in Fig. 3C, the peak at $1205 \mathrm{~cm}^{-1}$ was attributed to the stretching vibration of $\mathrm{C}-\mathrm{F}$ and the peak at $1120 \mathrm{~cm}^{-1}$ was attributed to the stretching vibration of $\mathrm{C}-\mathrm{O}-\mathrm{C}$. The appearance of the above characteristic peaks in PGA-PTX proved the structure of PGA-PTX. As shown in Fig. 3E, the observed absorbance bands of FA at $3117 \mathrm{~cm}^{-1}$, $833 \mathrm{~cm}^{-1}$ and $763 \mathrm{~cm}^{-1}$ were characteristic for the bending vibration of $\mathrm{C}-\mathrm{H}$ on benzene ring. The stretching vibration at $1410 \mathrm{~cm}^{-1}$ and $1131 \mathrm{~cm}^{-1}$ were attributed to the $\mathrm{C}-\mathrm{N}$ in FA. The peaks $\left(3062,2937,1653,1548,1404,1120,826,770 \mathrm{~cm}^{-1}\right)$ in PGA-FA indicated successful synthesis of PGA-FA. Therefore, the results confirmed successful synthesis of PGA-PTX, PGAGEM and PGA-FA.

In ${ }^{1} \mathrm{H}$ NMR spectra of PGA-PTX (Fig. 3B), the peaks of PGA or PTX appeared at $1.02 \mathrm{ppm}\left(-\mathrm{CH}_{3}-\mathrm{a}\right), 4.49 \mathrm{ppm}$ (-COCHNH-b), $7.33 \mathrm{ppm}$ (-Ph-c) and 7.89-7.68 ppm (-Ph-d). In ${ }^{1} \mathrm{H}$ NMR spectra of PGA-GEM (Fig. 3D), the peaks of PGA or GEM appeared at $7.45 \mathrm{ppm}$ (-Ph-f), $5.99 \mathrm{ppm}$ (-CF $\left.\mathrm{CF}_{2} \mathrm{CHO}-\mathrm{e}\right), 4.49 \mathrm{ppm}$ (-COCHNH-b) and $0.97 \mathrm{ppm}\left(-\mathrm{CH}_{3}-\mathrm{a}\right)$. In ${ }^{1} \mathrm{H}$ NMR spectra of PGA-FA (Fig. 3F), the peaks of PGA or FA appeared at $7.48 \mathrm{ppm}$ (-Ph-h), 6.66 ppm (-Ph-g), 4.49 ppm (-COCHNH-b), 0.94 ppm 

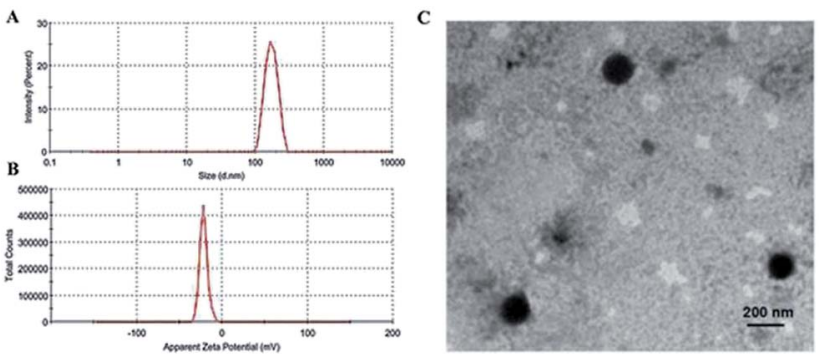

Fig. 4 Morphology of MF-FA NPs. (A) Size distributions. (B) Zeta potential distributions. (C) TEM of MF-FA NPs.

$\left(-\mathrm{CH}_{3}-\mathrm{a}\right)$. Consequently, PTX, GEM and FA were successfully conjugated with the carboxylic acid of PGA.

\subsection{Preparation and characterization of functionalized nanoparticles}

The particle size and zeta potential of different proportions were shown in Fig. S4. $\dagger$ The result indicated that the ratio of $1.5: 1$ exhibited the smallest particle size and suitable zeta potential compared with other ratios. So we chose the ratio of $1.5: 1$ to carry out the studies.

As summarized in Fig. 4A, the average particle size of MF-FA NPs was about $170 \mathrm{~nm}$, with a PDI of 0.232 . In addition, the zeta potential of the MF-FA NPs was $-21.60 \mathrm{mV}$ (Fig. 4B). The particle size of MF-FA NPs observed by TEM was about $160 \mathrm{~nm}$ (Fig. 4C), which was smaller than the results determined by DLS. The particle size determined by DLS represented the hydrodynamic diameter, whereas the results obtained by TEM were the collapsed nanoparticles after water evaporation. ${ }^{32}$ Moreover, the drug loading (DL) was measured to further evaluate the nanoparticles. The DL of PGA-PTX, PGA-GEM and PGA-FA were $18.6 \%, 19.56 \%$ and $19.4 \%$ respectively, and the experimental results were as expected as theory.

\subsection{In vitro drug release of PTX and GEM}

Effective anticancer carriers should provide targeting drug release and accumulation at tumor site and subsequently lead to satisfactory therapeutic results. Therefore, in vitro drug release profiles for MF-FA NPs were investigated in different environments (pH 5.5 and 7.4), as shown in Fig. 5. The cumulative PTX and GEM release amount at pH 5.5 were remarkably higher than $\mathrm{pH} 7.4$, indicating that the release of drug was
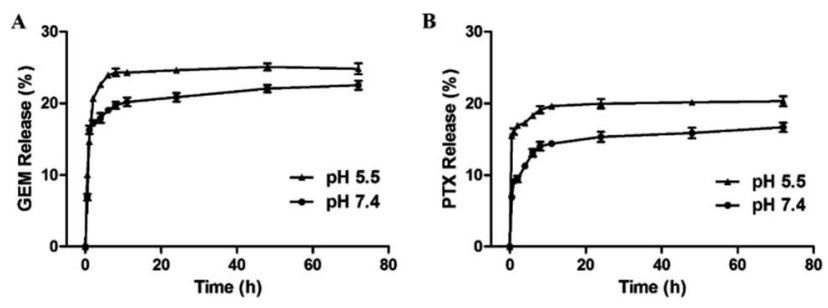

Fig. 5 Release profiles of GEM (A) and PTX (B) from MF-FA NPs in PBS $(\mathrm{pH} 5.5$ and $\mathrm{pH} 7.4$, respectively) containing $0.5 \%(\mathrm{v} / \mathrm{v})$ of Tween 80 . affected by $\mathrm{pH}$ condition, which could be attributed to the easy cleavage of ester bonds in acidic environments. In addition, the nanoparticles presented a rapid drug release in the first 12 hours, especially in $\mathrm{pH}$ 5.5. According to recent reports, for cancer treatment, it would be more desirable to achieve rapid and sufficient drug release once the carriers reach at the tumor site to obtain better therapeutic efficacy. Hence, the above results illustrated that MF-FA NPs exhibited a typical $\mathrm{pH}$ dependent and rapid drug release pattern, which would facilitate selective drug release within tumor cells.

\subsection{Stability of nanoparticles}

The stability of nanoparticles is crucial to their applications, such as long-term storage stability, prolonged biological activities at tumor site and circulation in body. As depicted in Fig. 6, no significant changes were detected in terms of particle size, zeta potential and PDI within $96 \mathrm{~h}$, indicating that the nanoparticles would remain stable at room temperature at least for up to four days.

\subsection{Hemolysis study}

The hemolysis study was carried out to evaluate the biocompatibility of nanoparticles. A hemolytic percentage less than $2 \%$ is considered as non-hemolytic and $2-5 \%$ are slightly hemolytic and more than $5 \%$ is hemolytic, according to ASTM F756-13 standard for nanomaterials. Distilled water caused significant damage of red blood cells (Fig. 7), and saline did not induce substantial hemolysis. In contrast, the hemolytic percentage of MF-FA NPs and MF NPs were all less than 2\% when both nanoparticles were tested from 2 to $10 \mathrm{mg} \mathrm{mL}^{-1}$ (Fig. 7), which indicated good safety for the nanomaterials.

\subsection{Study of antiproliferative ability}

The antiproliferative ability of drug-loaded MF-FA NPs, MF NPs and pure drug (PTX : GEM = $1: 1$ ) were evaluated using a CCK-8 assay on breast cancer cells 4T1. As shown in Fig. 8A, FA modified MF-FA NPs exhibited a better proliferation inhibition to $4 \mathrm{~T} 1$ cells with the concentration of 10 and $400 \mathrm{ng} \mathrm{mL}^{-1}$ than MF NPs after incubation for $72 \mathrm{~h}$. The enhanced proliferation inhibition to $4 \mathrm{~T} 1$ cells was owing to the folic acid specific targeting in the nanoparticles. However, other concentration of NPs exhibited no significant differences between MF-FA NPs and MF NPs perhaps due to the experimental errors. The results demonstrated that FA enhanced cellular uptake of the nanoparticles.
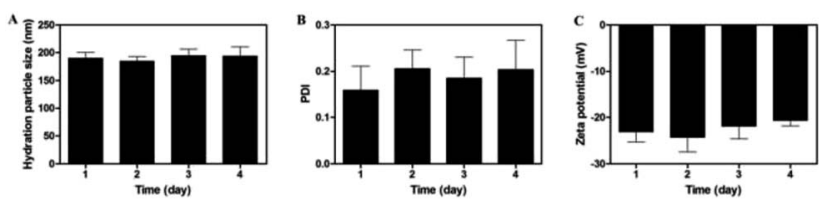

Fig. 6 Hydration particle size (A), PDI (B) and zeta potential (C) of MFFA NPs in four days at room temperature. 


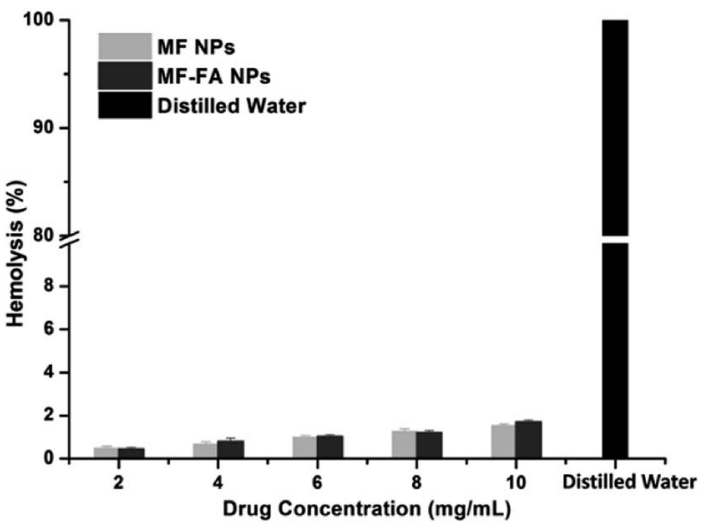

Fig. 7 Hemolysis of red blood cells after incubation with MF NPs, MFFA NPs and distilled water.
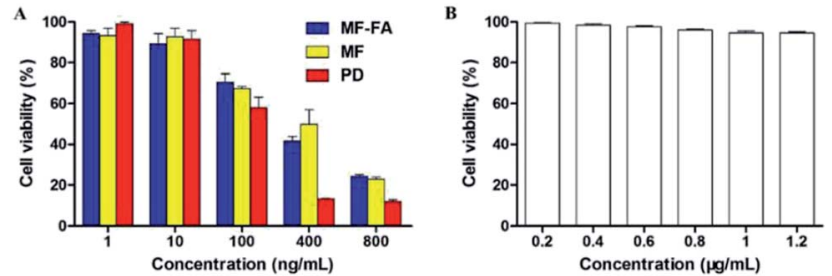

Fig. 8 Cell proliferation assessments using the CCK- 8 assay. (A) The proliferation inhibition of MF-FA NPs, MF NPs and pure drug to 4T1 cells at $72 \mathrm{~h}$. (B) The proliferation inhibition of blank NPs to 4T1 cells at $72 \mathrm{~h}$.

Besides, pure drug showed higher inhibition against 4T1 cells than the two nanoparticles due to the direct transportation into cells. While for the MF-FA NPs and MF NPs, the drugs were slowly released and the absorption by cells was delayed as previously reported in the vitro drug release study. ${ }^{33}$

In low concentration ( $1 \mathrm{ng} \mathrm{mL}^{-1}$ ), nanoparticles were enriched around cancer cells and sustained release drugs. Pure drug was dispersed in the culture solution and led to the lower inhibition against $4 \mathrm{~T} 1$ cells than the two NPs. The results in Fig. 8B showed that blank carriers did not exhibit proliferation inhibition to $4 \mathrm{~T} 1$ cells, which further illustrated that drugloaded NPs had high biocompatibility.

\subsection{In vivo antitumor efficacy}

The antitumor efficacies of MF-FA NPs, MF NPs and pure drug were evaluated in breast tumor bearing mice. As shown in Fig. 9A, in the control group, tumors quickly and continuously grew and the average tumor volume on day 21 was up to 1900 $\mathrm{mm}^{3}$ approximately. In other three groups, the curves of tumor volume were increased more slowly than the control group in 21 days. Compare with the pure drug group, the MF NPs group had a significant anti-tumor effect. Furthermore, the antitumor activity of FA-loaded NPs (MF-FA NPs) was more effective than FA-free NPs (MF NPs) due to the high expression of FA receptor in cancer cells. The results indicated that the targeting moiety FA of MF-NPs was able to effectively deliver PTX and GEM. In addition, the body weight of mice did not show significant loss
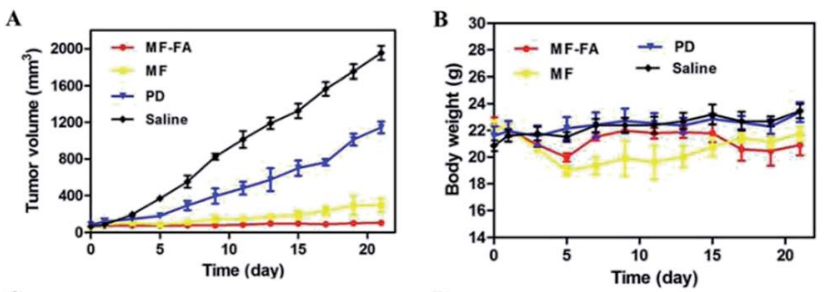

C
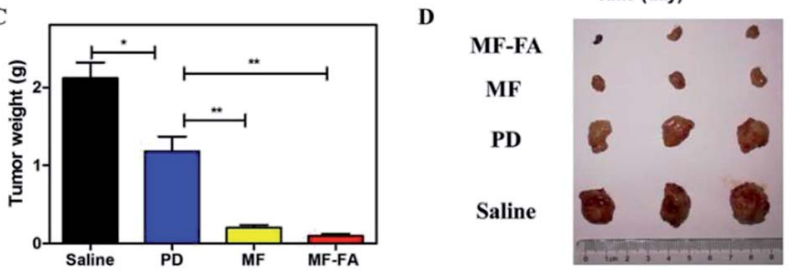

Fig. 9 In vivo anti-tumor effects of breast cancer 4T1 cell-induced BALB/c mice. (A) Tumor growth curves. (B) Body weights of the mice. (C) Average tumor weight after the study. (D) Images of tumor tissues. Data were shown as mean $\pm \mathrm{SD},(n=3)$. Significant difference from control: $* P<0.05, * * P<0.01, * * * P<0.001$.

in the duration of the study (Fig. 9B), indicating satisfactory safety of the testing nanoparticles. The tumor weights of NPs groups were significantly reduced compared with saline group (Fig. 9C and D) and the average weights of tumor in MF-FA NPs, MF NPs, PD and control groups were $0.09 \mathrm{~g}, 0.20 \mathrm{~g}, 1.18 \mathrm{~g}$ and $2.12 \mathrm{~g}$, respectively. There were significant differences $(p<$ 0.001 ) between saline and NPs groups.

Consequently, these results had successfully demonstrated the therapeutic efficacies of these nanocarriers at the tumor site. MF-FA NPs thus had significant potential as promising nano-platforms for targeting delivery of dual chemotherapy agents and effective tumor treatment method.

\section{Conclusions}

In summary, we successfully fabricated a multi-functional FA targeting nanocarrier delivery system for co-delivery of hydrophilic chemotherapeutic drug GEM and hydrophobic PTX for the breast cancer treatment. The intelligent nanoparticles demonstrated outstanding stability and better biocompatibility. Additionally, the nanoparticles presented favorable tumor targeting capability via a folate receptor-mediated endocytosis and rapidly released drug in the acidic tumor microenvironment, thereby increasing the efficacy of anticancer drugs in vivo. Therefore, it was expected that the multi-functional MF-FA NPs would establish a promising platform for combination therapy to improve therapeutic efficacy.

\section{Conflicts of interest}

The authors declare that they have no competing interests.

\section{Acknowledgements}

This work was supported by the following grants: the National Natural Science Foundation of China (21877061), Natural Science Foundation of Jiangsu Province (BK20171448), National 
and Local Joint Engineering Research Center of Biomedical Functional Materials and Priority Academic Program Development of Jiangsu Higher Education Institutions (PAPD).

\section{References}

1 A. Jemal, F. Bray, M. M. Center, J. Ferlay, E. Ward and D. Forman, Global cancer statistics, Ca-Cancer J. Clin., 2011, 61(2), 69-90.

2 J. Xie, Z. H. Tao, J. Zhao, T. Li, Z. H. Wu, J. F. Zhang, et al., Glucose regulated protein 78 (GRP78) inhibits apoptosis and attentinutes chemosensitivity of gemcitabine in breast cancer cell via AKT/mitochondrial apoptotic pathway, Biochem. Biophys. Res. Commun., 2016, 474(3), 612-619.

3 B. B. S. Zhou and J. Bartek, Targeting the checkpoint kinases: chemosensitization versus chemoprotection, Nat. Rev. Cancer, 2004, 4(3), 216-225.

4 S. N. Dorman, K. Baranova, J. H. M. Knoll, et al., Genomic signatures for paclitaxel and gemcitabine resistance in breast cancer derived by machine learning, Mol. Oncol., 2015, 10(1), 85-100.

5 L. Jiang, Y. S. Wang and J. X. Guo, Synergistic drug combinations tend to improve therapeutically relevant selectivity, Nat. Biotechnol., 2009, 27(7), 659-666.

6 L. B. Saltz, S. Clarke, E. D. Rubio, et al., Bevacizumab in combination with oxaliplatin-based chemotherapy as firstline therapy in metastatic colorectal cancer: a randomized phase III study, J. Clin. Oncol., 2008, 26(12), 2013-2019.

7 R. S. Herbst, G. Giaccone, J. H. Schiller, et al., Gefitinib in combination with paclitaxel and carboplatin in advanced non-small-cell lung cancer: a phase III trial-INTACT 2, $J$. Clin. Oncol., 2004, 22(5), 785-795.

8 G. Giaccone, R. S. Herbst, C. Manegold, et al., Gefitinib in Combination With Gemcitabine and Cisplatin in Advanced Non-Small-Cell Lung Cancer: A Phase III Trial-INTACT 1, J. Clin. Oncol., 2004, 22(5), 777-784.

9 V. T. Devita Jr, R. C. Young and G. P. Canellos, Combination versus single agent chemotherapy: A review of the basis for selection of drug treatment of cancer, Cancer, 1975, 35(1), 98-110.

10 M. A. Shah and G. K. Schwartz, The relevance of drug sequence in combination chemotherapy, Drug Resist. Updates, 2000, 3(6), 335-356.

11 M. Ferrari, Cancer nanotechnology: opportunities and challenges, Nat. Rev. Cancer, 2005, 5(3), 161-171.

12 L. D. Mayer, T. O. Harasym, P. G. Tardi, et al., Ratiometric dosing of anticancer drug combinations: controlling drug ratios after systemic administration regulates therapeutic activity in tumor-bearing mice, Mol. Cancer Ther., 2006, 5(7), 1854-1863.

13 G. F. V. Ismael, D. D. Rosa, M. S. Mano and A. Awada, Novel cytotoxic drugs: old challenges, new solutions, Cancer Treat. Rev., 2008, 34(1), 81-91.

14 H. Popilski, E. Abtew, S. Schwendeman, et al., Efficacy of paclitaxel/dexamethasone intra-tumoral delivery in treating orthotopic mouse breast cancer, J. Controlled Release, 2018, 10, 279-286.
15 X. Q. Li, H. Y. Wen, H. Q. Dong, et al., Self-assembling nanomicelles of a novel camptothecin prodrug engineered with a redox-responsive release mechanism, Chem. Commun., 2011, 47(30), 8647-8649.

16 S. P. Yin, J. Huai, X. Chen, et al., Intracellular delivery and antitumor effects of a redox-responsive polymeric paclitaxel conjugate based on hyaluronic acid, Acta Biomater., 2015, 26, 274-285.

17 A. K. Anal, A. Tobiassen, J. Flanagan and H. Singh, Preparation and characterization of nanoparticles formed by chitosan-caseinate interactions, Colloids Surf., B, 2008, 64(1), 104-110.

$18 \mathrm{~J}$. Khandare and T. Minko, Polymer-drug conjugates: Progress in polymeric prodrugs, Prog. Polym. Sci., 2006, 31(4), 359-397.

19 R. Namgung, Y. M. Lee, J. Kim, et al., Poly-cyclodextrin and poly-paclitaxel nano-assembly for anticancer therapy, Nat. Commun., 2014, 5, 3702-3714.

20 K. Cho, X. Wang, S. Nie, Z. Chen and D. M. Shin, Therapeutic nanoparticles for drug delivery in cancer, Clin. Cancer Res., 2008, 14(5), 1310-1316.

21 L. Ma, M. Kohli and A. Smith, Nanoparticles for Combination Drug Therapy, ACS Nano, 2013, 7(11), 95189525.

22 Z. Q. Pang, L. Feng, R. R. Hua, J. Chen, H. Gao, S. Q. Pan, et al., Lactoferrin-conjugated biodegradable polymersome holding doxorubicin and tetrandrine for chemotherapy of glioma rats, Mol. Pharm., 2010, 7(6), 1995-2005.

23 S. L. Dong, Y. X. Guo, Y. S. Duan, Z. M. Li, C. Wang, L. N. Niu, et al., Co-delivery of paclitaxel and gemcitabine by methoxy poly(ethylene glycol)-poly(lactide-coglycolide)-polypeptide nanoparticles for effective breast cancer therapy, AntiCancer Drugs, 2018, 29(7), 637-645.

24 W. Gao, B. Xiang, T. T. Meng, F. Liu and X. R. Qi, Chemotherapeutic drug delivery to cancer cells using a combination of folate targeting and tumor microenvironment-sensitive polypeptides, Biomaterials, 2013, 34(16), 4137-4149.

25 L. Bildstein, C. Dubernet and P. Couvreur, Prodrug-based intracellular delivery of anticancer agents, Adv. Drug Delivery Rev., 2011, 63(1), 3-23.

26 M. Prabaharan, J. J. Grailer, S. Pilla, D. A. Steeber and S. Q. Gong, Amphiphilic multi-arm-block copolymer conjugated with doxorubicin via $\mathrm{pH}$-sensitive hydrazone bond for tumor-targeted drug delivery, Biomaterials, 2009, 30(29), 5757-5766.

27 J. J. Lin, J. S. Chen, S. J. Huang, J. H. Ko, Y. M. Wang, T. L. Chen, et al., Folic acid-Pluronic F127 magnetic nanoparticle clusters for combined targeting, diagnosis, and therapy applications, Biomaterials, 2009, 30(28), 51145124 .

$28 \mathrm{Y}$. J. Lu and P. S. Low, Folate-mediated delivery of macromolecular anticancer therapeutic agents, Adv. Drug Delivery Rev., 2002, 54(5), 675-693.

29 L. Zhang, F. X. Gu, J. M. Chan, A. Z. Wang, R. S. Langer and O. C. Farokhzad, Nanoparticles in medicine: therapeutic 
applications and developments, Clin. Pharmacol. Ther., 2008, 83(5), 761-769.

30 I. Noh, H. O. Kim, J. Choi, Y. Choi, D. K. Lee, Y. M. Huh, et al., Co-delivery of paclitaxel and gemcitabine via CD44targeting nanocarriers as a prodrug with synergistic antitumor activity against human biliary cancer, Biomaterials, 2015, 53, 763-774.

31 L. P. Gao, H. Wang, L. J. Nan, T. Peng, L. Sun, J. G. Zhou, et al., Erythrocyte Membrane-Wrapped $\mathrm{pH}$ Sensitive
Polymeric Nanoparticles for Non-Small Cell Lung Cancer Therapy, Bioconjugate Chem., 2017, 28(10), 2591-2598.

32 L. P. Gao, L. F. Gao, M. X. Fan, Q. L. Li, J. Y. Jin, J. Wang, et al., Hydrotropic polymer-based paclitaxel-loaded selfassembled nanoparticles: preparation and biological evaluation, RSC Adv., 2017, 7(53), 33248-33256.

33 Y. Jin, L. Song, Y. Su, L. J. Zhu, Y. Pang, F. Qiu, et al., Oxime linkage: a robust tool for the design of $\mathrm{pH}$-sensitive polymeric drug carriers, Biomacromolecules, 2011, 12, 34603468. 